\title{
ANÁLISIS DE LA DISPONIBILIDAD DE HÁBITAT ADECUADO PARA EL LOBO (CANIS LUPUS) EN CATALUÑA Y EN LOS PIRINEOS ORIENTALES
}

\section{Habitat suitability analysis for the wolf (Canis lupus) in Catalonia and the Eastern Pyrenees}

\author{
Carla Garcia-Lozano, Josep Pintó y Josep Vila Subirós \\ Departamento de Geografía / Instituto de Medio Ambiente. Universitat de Girona. Pl. Ferrater Mora, 1. 17071 Girona. \\ alracgalo@gmail.com,josep.pinto@udg.edu,josep.vila@udg.edu \\ * Autor corresponsal: alracgalo@gmail.com
}

Recibido: 14-05-2015. Aceptado: 24-07-2015. Fecha de publicación on-line: 20/10/2015.

Citation / Cómo citar este artículo: Garcia-Lozano, C., Pintó, J. y Vila Subirós, J. (2015). Análisis de la disponibilidad de hábitat adecuado para el lobo (Canis lupus) en Cataluña y en los Pirineos orientales. Pirineos, 170, e014. doi: http://dx.doi. org/10.3989/Pirineos.2015.170007

RESUMEN: Después de décadas de ausencia del lobo (Canis lupus) en los Pirineos, desde finales de los años noventa se han detectado algunos ejemplares solitarios en el sector oriental de la Cordillera. El establecimiento definitivo del lobo dependerá de varios factores, entre los que cabe destacar la disponibilidad de hábitat adecuado, las medidas de conservación de la especie y la conectividad ecológica del paisaje. El presente estudio analiza la disponibilidad de hábitat para el lobo en Cataluña y la vertiente francesa de los Pirineos orientales mediante la elaboración de un mapa de coste de fricción o matriz de permeabilidad para la especie en cuestión, basado en siete parámetros de tipo territorial y ambiental. Posteriormente, se han seleccionado los espacios más óptimos para el lobo y se han delimitado las zonas geográficas más susceptibles para albergar el cánido en un futuro próximo. Los resultados del análisis confirman que un tercio del área estudiada constituye un hábitat óptimo para el lobo y alrededor del $90 \%$ de estos espacios cuentan con unas dimensiones suficientes para albergar poblaciones estables de lobos.

PALABRAS CLAVE: Lobo (Canis lupus); adecuación del hábitat; disponibilidad de hábitat; mapa de coste de fricción; matriz de permeabilidad; Pirineos; Prepirineos.

\begin{abstract}
After decades of absence of the wolf (Canis lupus) in the Pyrenees, since the end of the decade of the nineties some solitary specimens were detected in the eastern half of the mountains. Wolf establishment will depend on several factors, among which it is worth mentioning specie conservation measures, habitat availability and landscape's ecological connectivity. The present study analyzes the availability of suitable habitat for wolf in Catalonia and the French side of oriental Pyrenees, by means of a map of resistance surfaces for the predator based on seven territorial and environmental parameters. Afterwards, the most suitable zones have been selected and have been delimited those geographic areas which could be home for wolves in a near future. The results of the analysis confirm that one-third of the studied area constitutes an optimal habitat for wolf and around $90 \%$ of these spaces are provided with enough dimensions to hold stable wolves' populations.
\end{abstract}

KEYWORDS: Wolf (Canis lupus); habitat suitability; habitat availability; resistance surfaces; Pyrenees; Pre-Pyrenees. 


\section{Introducción}

A pesar de los acuerdos adoptados en la Cumbre de Río de 1992 por parte de la comunidad internacional en favor de la conservación de la biodiversidad, y las sucesivas revisiones de los objetivos a alcanzar, entre ellos el conseguir una significativa reducción de la tasa de extinciones para el año 2010, lo cierto es que la biodiversidad no ha cesado de disminuir a lo largo de las últimas décadas (Butchart et al., 2010). Sin embargo, las políticas orientadas a la conservación de las especies y los ecosistemas han sido un instrumento clave para evitar que la pérdida de biodiversidad fuera aún mayor y en el caso de algunas especies lograr revertir una tendencia que las dirigía a su extinción (Hoffmann et al., 2010).

En Europa las políticas de conservación de los grandes carnívoros han probado su eficacia, mostrando que el modelo de coexistencia entre las poblaciones de carnívoros y las comunidades humanas es factible y que en el caso del lobo presenta mejores resultados, en términos de número absoluto de individuos, que el modelo de separación, adoptado en los Estados Unidos y en muchos países en vías de desarrollo (Chapron et al., 2014).

La emergencia de los movimientos ambientalistas, ya en los años setenta, estuvo en la base de las acciones legislativas posteriores orientadas a la preservación de la biodiversidad, como por ejemplo el Convenio de Berna (1979) o la Directiva Hábitats (1992). Como consecuencia, los grandes carnívoros existentes aún en Europa (el oso pardo -Ursus arctos-, el lince -Lynx-, el glotón -Gulo gulo- y por supuesto el lobo -Canis lupus-) gozan de algún tipo de protección en el conjunto de países de la Unión Europea.

La implementación de redes de espacios naturales protegidos, junto con los cambios socioeconómicos que han afectado el espacio rural europeo en las últimas décadas, ha supuesto una mejora de la calidad de los hábitats, hecho que ha beneficiado la recuperación de las poblaciones de grandes carnívoros. El abandono de las actividades agrosilvopastorales tradicionales y el incremento de los ungulados salvajes ha propiciado su retorno a muchos paisajes humanizados de donde había desaparecido varias décadas atrás (Gortázar et al., 2000; Echergaray \& Vilà, 2010).

El lobo, antes de empezar su proceso de rarificación, se encontraba extendido a lo largo de todo el Hemisferio Norte allí dónde la presencia de ungulados en estado salvaje permitía su supervivencia (Mech, 1995). Los ataques del predador al ganado fueron el desencadenante del conflicto entre el lobo y los humanos, lo que conllevó la caza indiscriminada del cánido, sobre todo en los siglos XIX y $\mathrm{XX}$, disminuyendo drásticamente sus efectivos a escala global llegando a ser exterminadas numerosas poblaciones locales en distintos países de Europa (Núñez-Quirós et al., 2007).

A mediados del siglo XIX se produjo una severa regresión de las poblaciones españolas, un proceso que se inició en el extremo noreste extendiéndose por la costa mediterránea y progresivamente hacia el interior, llegán- dose a alcanzar el límite de la extinción a principios de los años 70 del siglo pasado (Rico \& Torrente, 2000; Núñez-Quirós et al., 2007). A partir de los años 80 se implantaron medidas de protección que propiciaron un incremento de las poblaciones en el noroeste peninsular y al sur del río Duero, aunque en este último sector las poblaciones todavía son poco numerosas y aisladas entre ellas.

El lobo es un animal muy generalista en cuanto a la selección de hábitat, ya que presenta una gran adaptabilidad a las condiciones ambientales y los recursos del territorio. Se pueden encontrar lobos bajo cualquier clima y ambiente, adoptando dietas diversas, desde las llanuras cerealistas deshabitadas hasta zonas considerablemente humanizadas (Blanco \& Cortés, 2002; Landry, 2004; Cayuela, 2004; Eggermann et al., 2011).

De hecho, la abundancia de presas naturales en estado salvaje para el lobo, principalmente el jabalí (Sus scrofa) y el corzo (Capreolus capreolus), es un factor clave para la repoblación del cánido. Además, este predador juega un papel fundamental en la regulación de la población de estos ungulados cuyos crecimientos continuos están ocasionando notables problemas en relación a los cultivos o a la seguridad vial. En este sentido, cabe destacar que las compensaciones económicas destinadas a los daños ocasionados por el jabalí son mucho más elevadas que los posibles daños derivados de los ataques del lobo al ganado (González-Prat, 2002).

En la Península Ibérica hay poblaciones estables de lobo ibérico (Canis lupus signatus) en el norte de Portugal, Cantabria, Galicia, Asturias, La Rioja, el País Vasco, Castilla y León y en menor proporción en Sierra Morena, Castilla La Mancha y Extremadura (Salvatori \& Linnell, 2005; Echergaray \& Vilà, 2010). Además, en los últimos años se han recogido muestras que evidencian la presencia de lobos solitarios cerca del límite entre el País Vasco y Navarra (Sáenz de Buruaga et al., 2000), en el norte de Aragón (Gortázar et al., 2000) y en el norte de Cataluña (Lampreave et al., 2011).

En Cataluña la población de lobos disminuyó drásticamente entre los siglos XVIII y XIX cuando el predador se vio obligado a atacar los rebaños domésticos debido a un descenso brusco en las poblaciones de grandes ungulados salvajes, resultado de una descontrolada cacería humana (Manent, 2004). Se conservan además testimonios documentales de las recompensas ofrecidas por las autoridades por la caza del lobo, las cuales muestran cómo el número de capturas va en descenso entre los siglos XVIII y XIX debido a la disminución en efectivos de su población (Grau et al, 1990). La reducción y fragmentación de las masas forestales y la utilización de estricnina, armas de fuego y trampas en las campañas de exterminio del lobo fueron el desencadenante de la desaparición de este mamífero en Cataluña y en muchas otras regiones del continente europeo.

Después de un largo tiempo sin rastro del lobo, a principios del siglo actual se inició la recuperación de este depredador en ciertas partes del sector oriental de los Pirineos y Prepirineos. Las primeras muestras que confirman 
su retorno se recogieron en el Parque Natural del CadíMoixeró en el año 2000, aunque no fue hasta 2004 cuando se analizaron las heces recogidas entonces y desde el Departamento de Medio Ambiente de la Generalitat de Cataluña se anunció oficialmente el retorno del animal en Cataluña (Lampreave et al., 2011).

Las muestras analizadas hasta el año 2011 confirman que un mínimo de trece lobos distintos han pasado por el territorio catalán, doce machos y una hembra (Lampreave et al., 2011). El cánido ya se ha observado en distintas comarcas catalanas situadas alrededor del macizo del Cadí como el Ripollès, el Solsonès, la Cerdanya, l'Alt Urgell, el Berguedà, $\mathrm{y}$ ha realizado alguna incursión puntual en el Moianès y en Osona. A pesar de ello, en los últimos tres años el flujo de lobos solitarios en el Pirineo, se ha reducido a uno o dos ejemplares según los datos del programa de seguimiento del lobo del Servicio de Biodiversidad y Protección de los Animales del Departamento de Agricultura, Ganadería, Pesca, Alimentación y Medio Natural de la Generalitat de Cataluña (David Camps, com. pers.).

Por otro lado, en la vertiente francesa las muestras evidencian la presencia de cuatro individuos diferentes de lobo en los macizos de Madres, Carlit-Peric y Canigó, entre los cuales uno resultó ser hembra (Lampreave et al., 2011). La presencia de hembras lobo en los Pirineos orientales abre la posibilidad de formación de al menos una pareja reproductiva en la zona.

En la mitad oriental de los Pirineos, el lobo no llegó desde la cornisa Cantábrica o el sistema Ibérico como cabía esperar que sucediera, sino que lo hizo desde el noreste, procedente de las poblaciones de lobo italianas (Canis lupus italicus). Desde que el gobierno italiano protegió la especie a mediados de los años setenta, las poblaciones de lobo se han extendido progresivamente desde el Parque Nacional de los Abruzzos, situado en los Apeninos centrales, hasta el Parque Natural de Mercantour, en los Alpes franceses, donde llegaron a principios de los años 90. A su vez, estas poblaciones se dispersaron rápidamente hasta llegar en 1999 al Macizo de Madres, en los Pirineos orientales franceses, desde donde meses más tarde cruzaron la frontera catalana (González-Prat, 2002; Lampreave et al., 2011).

De acuerdo con la Directiva Hábitats, el lobo es una especie prioritaria de interés comunitario en toda Europa, excepto las poblaciones españolas localizadas al norte del río Duero, las poblaciones del norte de Grecia y las de toda Finlandia. En España este animal está considerado por el Catálogo Nacional de Especies Amenazadas como una especie de caza al norte del Duero, pero está estrictamente protegida en Extremadura, Andalucía y Castilla La Mancha. En Cataluña carece de protección estatal puesto que cuando se elaboró dicho catálogo, el lobo no estaba presente en la comunidad autónoma. En cambio, la Generalitat de Cataluña prevé incluir el depredador en la categoría de "en peligro de extinción" en el Catálogo de Fauna Amenazada de Cataluña, texto que actualmente se encuentra pendiente de aprobación. Por lo que respeta a la legislación francesa, el lobo es una especie protegida a escala nacional desde 1993, momento en que se detectó la presencia del cánido en los Alpes franceses (Fourli, 1999).

La situación geográfica de Cataluña permite que su territorio sea un punto de confluencia entre la subespecie de lobo propia de la Península Ibérica, Canis lupus signatus, y la subespecie originaria de los Apeninos italianos, $\mathrm{Ca}$ nis lupus italicus. Al norte del río Duero el lobo se considera una especie cinegética, lo que permite perseguir y matar indiscriminadamente a los individuos de dichas poblaciones. Por el contrario, las políticas de conservación y recuperación medioambiental llevadas a cabo en buena parte de Europa, sumadas a la protección de especies vulnerables, ha hecho posible una lenta pero constante recuperación del lobo en ciertas zonas del continente, sobre todo en Italia, Suiza y en el este y centro de Francia (Poulle et al., 1995). Por este motivo, es posible que el lobo siga penetrando en Cataluña desde Francia en lugar de hacerlo desde la Meseta.

La protección de las especies vulnerables es un factor clave para la recuperación de la biodiversidad. Ahora bien, el éxito o fracaso del restablecimiento de estas especies depende también de otros factores, como la disponibilidad de hábitat adecuado o el estado de la conectividad entre los fragmentos de hábitat disponibles.

En este trabajo se analiza la disponibilidad de hábitat potencial para el lobo en Cataluña y en la vertiente francesa de los Pirineos orientales con el objetivo de determinar si las condiciones ambientales son lo suficientemente adecuadas para la reproducción y el posterior establecimiento de la especie en el área de estudio. La movilidad y dispersión de cualquier organismo, lejos de estar influenciada por una lógica administrativa, está sujeta a las condiciones ambientales de los territorios que colonizan, por dicho motivo se ha optado por ampliar la zona de estudio más allá de los límites administrativos de Cataluña, puesto que las muestras genéticas recogidas hasta el momento en ambas laderas de los Pirineos determinan que se trata de los mismos individuos de lobo que divagan de la vertiente norte a la sur de la cordillera pirenaica (Lampreave et al., 2011). Así mismo, este análisis transfronterizo también incluye el estado de Andorra ya que su localización en la cordillera pirenaica lo hace susceptible de albergar lobos si las condiciones ambientales lo permiten.

Para analizar la disponibilidad de hábitat adecuado para la colonización del lobo se ha elaborado un mapa de adecuación del hábitat para el lobo mediante un conjunto de herramientas SIG. Esta técnica es de uso habitual, tanto en el análisis de la disponibilidad de hábitat (Suau, 2012; Velez-Liendo et al., 2014; Zeller et al., 2012) como para el desplazamiento de las especies a través de la matriz paisajística (Liu \& Li, 2008; Rabinowitz \& Zeller, 2010; Gurrutxaga et al., 2010), en un proceso metodológico similar al de la elaboración de los modelos de favorabilidad basados en parámetros ambientales (Acevedo et al., 2011). 


\section{Metodología}

\section{1. Área de estudio}

El ámbito territorial donde se enmarca el presente estudio transfronterizo es el conjunto del territorio catalán junto con la vertiente francesa de los Pirineos orientales y el pequeño estado de Andorra (Figura 1).

Dado que las muestras recogidas sobre la presencia del lobo en Cataluña indican que su área de distribución incluye ambas laderas de la cordillera pirenaica, se decidió ampliar en este análisis la zona de estudio más allá de los límites administrativos de Cataluña, de manera que se incluyeron también los territorios de Andorra y de la vertiente norte de la mitad oriental de la cordillera pirenaica (parte de los departamentos franceses del LanguedocRoussillon y el Midi-Pyrénées), abarcando en total una extensión aproximada de $49.000 \mathrm{~km}^{2}$.

Aunque la media de altitud del área objeto de estudio no es especialmente elevada (más del 75\% del área de estudio se encuentra por debajo de los 1.000 metros), las elevaciones del terreno son abundantes y su disposición favorece una gran diversidad ambiental. La gran cordillera de los Pirineos recorre de este a oeste la zona norte del área alcanzando las máximas altitudes en el centro de la cordillera o zona axial, destacando la Pica d'Estats (3.147 m). A su vez, los Prepirineos están compuestos por una serie de sierras extendidas en paralelo al eje axial, llegando a formar en la vertiente sur un relieve complejo constituido por macizos y sierras que llegan a alcanzar altitudes de más de 2.500 metros en la Sierra del Cadí. En la vertiente norte la altitud disminuye de forma muy abrupta de manera que los Prepirineos solo están representados por el macizo de les Corbières, que tiene en el Pic de Bugarach $(1.230 \mathrm{~m})$ su cima más elevada.

Así mismo, cabe destacar el Sistema Mediterráneo Catalán, un sistema compuesto por la Cordillera Prelitoral y Litoral, cadenas montañosas paralelas a la costa. El relieve del resto del territorio está compuesto por planicies y depresiones, entre las que cabe mencionar la Depresión Central, la Depresión Prelitoral, la Plana de l'Empordà y la Plana del Roselló, esta última situada en territorio francés.

Dada la disposición de los accidentes geográficos, la zona de estudio cuenta con una variedad notable de climas: clima mediterráneo litoral, clima mediterráneo con tendencia continental, clima submediterráneo, clima atlántico y clima de montaña. Así pues, la vegetación de la zona es propia de tres regiones biogeográficas distintas: predomina la vegetación mediterránea, aunque también abunda la eurosiberiana y en menor proporción la boreoalpina.

La gran variedad climática, vegetal y altitudinal provee las condiciones necesarias para albergar una gran diversidad faunística. De hecho, abunda el rebeco (Rupicapra pyrenaica), el corzo (Capreolus capreolus), el jabalí (Sus scrofa), la liebre (Lepus europaeus) y en menor medida el muflón (Ovis orientalis) y el ciervo (Cervus elaphus), todos ellos presas en estado salvaje para el lobo.
Por lo que se refiere a las cubiertas del suelo presentes en el área de estudio, la tipología predominante son los espacios agrarios (36\%) y los bosques (33\%), seguido de los matojos y herbazales (21\%), mientras que los espacios antrópicos ocupan menos del $4 \%$ y los pastos solamente el 3\% (ETC/SIA, 2013). Aunque los pastos ocupan un porcentaje bajo respeto a otras tipologías de usos del suelo, la ganadería extensiva es especialmente relevante en la zona del Pirineo y Prepirineo, donde se encuentran principalmente ganado equino y bovino, si bien también hay ganado ovino y caprino en menor proporción.

Los espacios forestales, que ocupan más del $50 \%$ del área, están mayormente ubicados en las principales elevaciones del terreno, justamente donde también se hallan la mayor parte de los espacios protegidos de notorio valor ecológico y paisajístico. En total, más del 35\% de la zona estudiada goza de alguna figura de protección.

Desde el punto de vista demográfico, el territorio catalán es un espacio densamente poblado $\left(236 \mathrm{hab} / \mathrm{km}^{2}\right.$ en 2014), si bien presenta un gran desequilibrio poblacional ya que la mayor parte de sus habitantes viven en la región metropolitana de Barcelona y en las zonas costeras, mientras que las áreas de montaña, como es el caso de los Pirineos, constituyen la zona más despoblada. En cambio, Andorra y el sector suroriental de Francia (los departamentos franceses de Ariège, Pyrénées-Orientales y la mitad sur de Haute-Garonne y Aude), al tratarse de regiones montañosas, cuentan con densidades de población bajas (164 hab/ $\mathrm{km}^{2}$ en 2014 y 104 hab $/ \mathrm{km}^{2}$ en 2012, respectivamente).

\subsection{Elaboración del mapa de adecuación del hábitat para el lobo}

Para obtener el mapa de adecuación del hábitat para el lobo se utilizó la técnica de análisis cartográfico digital conocida en lenguaje SIG como superposición ponderada. Esta técnica consiste en superponer las variables de forma ponderada que se considera ejercen mayor influencia sobre el hábitat del lobo a través del paisaje (Tabla 1) a fin de localizar aquellas zonas que presentan unos atributos más favorables para la colonización de dicha especie. El resultado que se obtiene es un mapa que diferencia entre las áreas que acumulan un mayor coste de adecuación: las más desfavorables, de las que acumulan un coste menor: las más favorables, de tal manera que se pueden obtener los emplazamientos más adecuados para el establecimiento del lobo.

Para ese fin, se han utilizado dos parámetros de tipo ambiental y territorial representados en total por 7 variables o indicadores. Dichos parámetros son: a) la disponibilidad de alimento, evaluada por las variables tipología de hábitat y proximidad a los recursos fluviales, y b) la presión antrópica, evaluada por los siguientes 5 indicadores: altitud, distancia a las áreas urbanas, distancia a las carreteras, distancia a las vías férreas y presencia de espacios naturales protegidos (Tabla 2). 


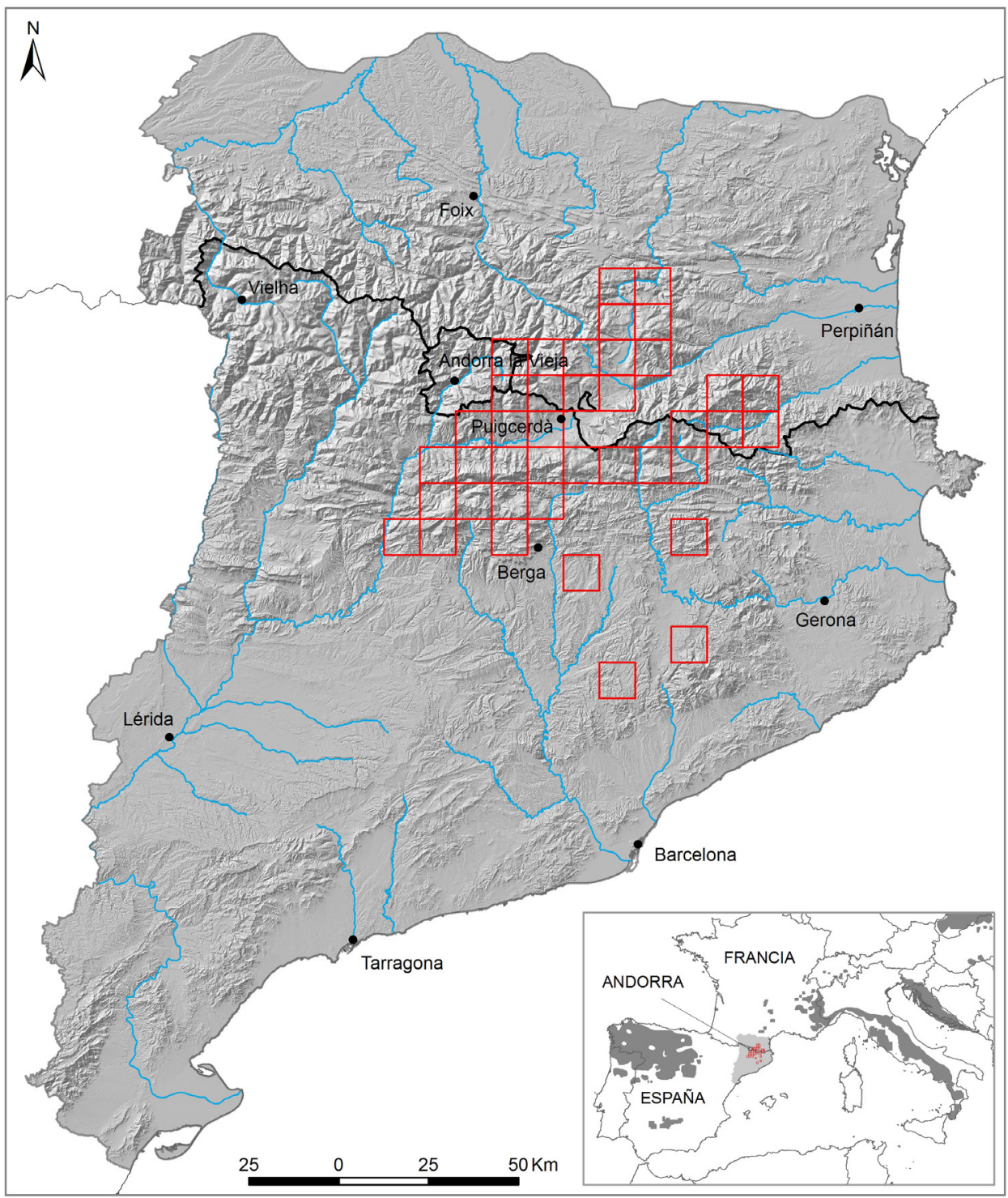

Figura 1: Localización del área de estudio y distribución del lobo en el sur de Europa. Las cuadrículas rojas indican presencia del lobo en algún momento desde 2003 hasta 2011. Fuente: Elaboración propia a partir de los datos sobre la presencia del lobo tomados de Salvatori, \& Linnell, 2005; Blanco et al., 2007 y Lampreavre et al., 2011.

Figure 1: Study area location and Wolf distribution in the southern Europe. Red squares indicate presence of wolf at some time since 2003 to 2011.

Los valores otorgados a cada variable y la ponderación de las capas se han obtenido a partir de datos publicados en revistas científicas sobre los hábitos del lobo (especificados más adelante al examinar cada una de las variables utilizadas), pudiéndose considerar este proceso metodológico dentro de los denominados como basado en opinión experta (Zeller et al., 2012).

Toda la información cartográfica se ha tratado con el programa ArcMap de ESRI. Se han trabajado los mapas con la proyección cartográfica UTM (Universal Transverse Mercator) usando el sistema de referencia ETRS89 (European Terrestrial Reference System 1989), UTM zona $31 \mathrm{~N}$, transformado en numerosas ocasiones la pro- yección cartográfica de las variables a fin de operar siempre con la misma proyección.

Las capas vectoriales, después de ser tratadas mediante un conjunto de herramientas SIG (buffer, clip, merge, euclidean distance...), fueron rasterizadas con una resolución de píxel de $30 \mathrm{~m}^{2}$. Posteriormente, las variables fueron debidamente reclasificadas mediante un valor de coste de adecuación determinado para el lobo (Tabla 2). Las distintas capas de información cartográfica se han ponderado, es decir, sumado y multiplicado por el peso otorgado, dando lugar a un mapa ráster que acumula los valores definitivos de coste de adecuación del hábitat para el lobo en cada píxel (Figura 2). 
Tabla 1: Procedencia de los datos utilizados en la elaboración del mapa de adecuación del hábitat para el lobo. Table 1: Data source used for the elaboration of the wolf habitat suitability map.

\begin{tabular}{|c|c|c|c|c|}
\hline Estado & Capa & Año & Fuente & Escala \\
\hline \multirow{10}{*}{ España } & Tipología de hábitats (Hábitats de Cataluña) & 2005 & $\begin{array}{l}\text { DTES (Departamento de Territorio y Sostenibilidad) } \\
\text { y UB }\end{array}$ & $1: 50.000$ \\
\hline & Red fluvial & 2004 & ACA (Agència Catalana de l'Aigua) & $1: 50.000$ \\
\hline & Red ferroviaria & 2007 & DTES & $1: 5.000$ \\
\hline & Red viaria & 2013 & DTES & $1: 5.000$ \\
\hline & Espacios de interés natural: & & \multirow{4}{*}{ DTES } & \\
\hline & Red Natura 2000 & 2013 & & $1: 50.000$ \\
\hline & PEIN (Plan de Espacios de Interés Natural) & 2013 & & $1: 25.000$ \\
\hline & Espacios naturales de protección especial & 2012 & & $1: 50.000$ \\
\hline & Áreas urbanas & 2005 & Elaboración propia a partir de la capa de hábitats & $1: 50.000$ \\
\hline & Mapa de elevaciones del terreno & 2014 & ICC (Institut Cartogràfic de Catalunya) & $1: 25.000$ \\
\hline \multirow{9}{*}{ Francia } & Tipología de hábitat (CORINE Land Cover) & 2006 & Agencia Europea de Medio Ambiente (AEMA) & $100 \mathrm{~m}^{2}$ \\
\hline & Red fluvial & 2014 & Open Street Map (OSM) & - \\
\hline & Red ferroviaria & 2014 & OSM & - \\
\hline & Red viaria & 2014 & OSM & - \\
\hline & \multicolumn{2}{|l|}{ Espacios de interés natural: } & & \\
\hline & Red Natura 2000 & 2011 & AEMA & $1: 100.000$ \\
\hline & Parques naturales regionales & - & $\begin{array}{l}\text { Ministerio de Ecología, Desarrollo Sostenible y } \\
\text { Energía (MEDDE) }\end{array}$ & - \\
\hline & Áreas urbanas & 2006 & Elaboración propia a partir de la capa de hábitats & $1: 100.000$ \\
\hline & Mapa de elevaciones del terreno & & & \\
\hline \multirow{7}{*}{ Andorra } & Tipología de hábitats (Hábitats de Andorra) & 2012 & Instituto de Estudios Andorranos & $1: 25.000$ \\
\hline & Red fluvial & 1995 & Instituto de Estudios Andorranos & $1: 5.000$ \\
\hline & Red viaria & 1995 & $\begin{array}{l}\text { Departamento de Medio Ambiente } \\
\text { (Gobierno de Andorra) }\end{array}$ & $1: 5.000$ \\
\hline & Espacios de interés natural: & & \multirow[b]{2}{*}{$\begin{array}{l}\text { Departamento de Medio Ambiente } \\
\text { (Gobierno de Andorra) }\end{array}$} & \multirow[b]{2}{*}{$1: 50.000$} \\
\hline & Parques naturales & 2013 & & \\
\hline & Áreas urbanas & 2012 & Elaboración propia a partir de la capa de hábitats & $1: 25.000$ \\
\hline & Mapa de elevaciones del terreno & 1995 & $\begin{array}{l}\text { Departamento de Medio Ambiente (Gobierno de } \\
\text { Andorra) }\end{array}$ & $5 \mathrm{~m}^{2}$ \\
\hline
\end{tabular}

Fuente: Elaboración propia.

Cabe destacar que el método utilizado de superposición ponderada de capas presenta algunos problemas y limitaciones, entre los que cabe destacar la interpolación de datos que resulta de este tipo de operaciones ráster (Franklin, 2009).

La escala de origen de las distintas capas de información también es un elemento a tener en cuenta, puesto que determina el detalle de la cartografía. En este sentido, la tipología de hábitats de Francia está elaborada a una escala muy general $(1: 100.000)$ en relación al resto del área de estudio (1:50.000 para Cataluña y 1:25.000 para Andorra) (Tabla 1), lo cual hace que en los mapas de adecuación del hábitat obtenidos se aprecie más detalle en la zona de Cataluña y Andorra en comparación con la parte francesa donde las teselas de hábitat aparecen más homogéneas (Figuras 2 y 3 ).

Una de las principales variables en el establecimiento del lobo en el territorio es la disponibilidad de alimento. Este factor depende en gran medida de la cubierta del suelo o tipología del hábitat. Estudios realizados sobre las poblaciones de lobos en paisajes humanizados han determinado que la densidad de lobos está directamente relacionada con la densidad de presas en estado salvaje (Llaneza et al., 2012), por lo cual los espacios forestales son especialmente valiosos. También las tierras cercanas a los campos de cultivo son un espacio buscado por el lobo, ya que en él abundan presas como el jabalí, actualmente en expansión. Por otra parte, los roquedos de montaña tam- 


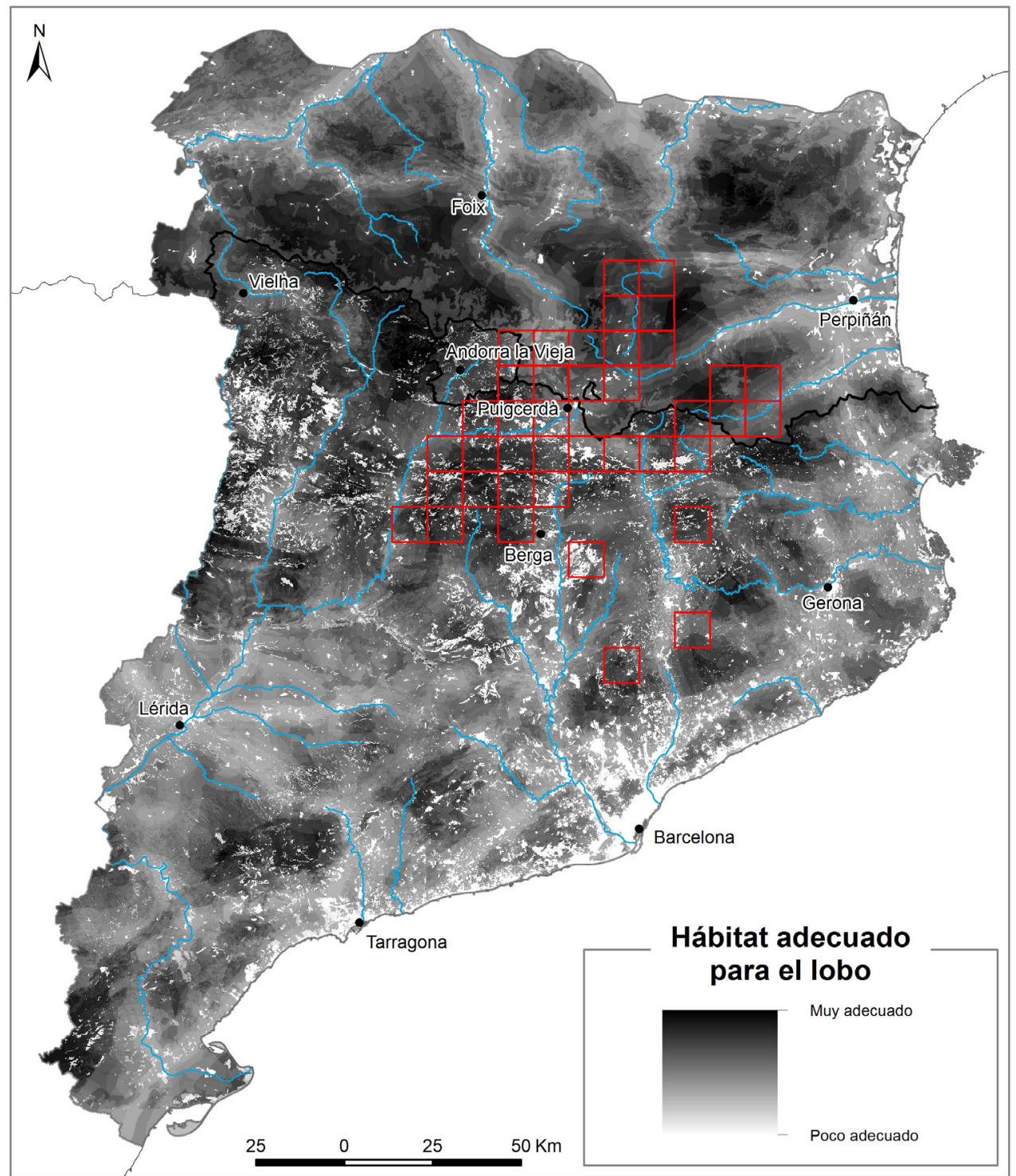

Figura 2: Grado de adecuación del hábitat del lobo. Las cuadrículas rojas indican presencia de lobo en algún momento desde 2003 hasta 2011. Fuente: Elaboración propia

Figure 2: Degree of wolf habitat suitability. Red squares indicate presence of wolf at some time since 2003 to 2011.

bién son utilizados por los lobos para cazar ungulados salvajes y establecer sus madrigueras en los orificios naturales presentes en el terreno (Landry, 2004).

Por tanto, en la asignación de valores de fricción a las distintas cubiertas del suelo se han considerado las cubiertas forestales y los prados y pastos como los hábitats más favorables para el lobo, seguidos de los matorrales y landas y los campos de cultivo, mientras que a las áreas urbanas y a los espacios antropizados se les ha asignado el mayor valor de coste de adecuación del hábitat (Tabla 2). Esta variable ha sido ponderada un $30 \%$ por encima del peso dado al resto de variables, al considerarse que ejerce una influencia superior sobre la disponibilidad de hábitat.
Los ríos constituyen un hábitat semipermeable para el lobo, ya que los puede atravesar con cierta facilidad en función del caudal y la fuerza de la corriente, y en ocasiones puede introducirse en ellos para perseguir a sus presas. Además el lobo necesita beber agua a diario por lo que debe utilizar los cursos de agua, pozas y manantiales, sobre todo en época de cría, cuando la hembra debe hidratarse constantemente para amamantar a los lobeznos (Landry, 2004). Por tanto es de suponer, dada la necesidad de disponer de agua diariamente, que no se alejará en demasía de dicho recurso. Dado que Vilà et al. (1990) han determinado, en un estudio sobre las poblaciones de lobos del noroeste de la Península Ibérica, que los movimientos diarios del cánido oscilan entre los 10 y los 


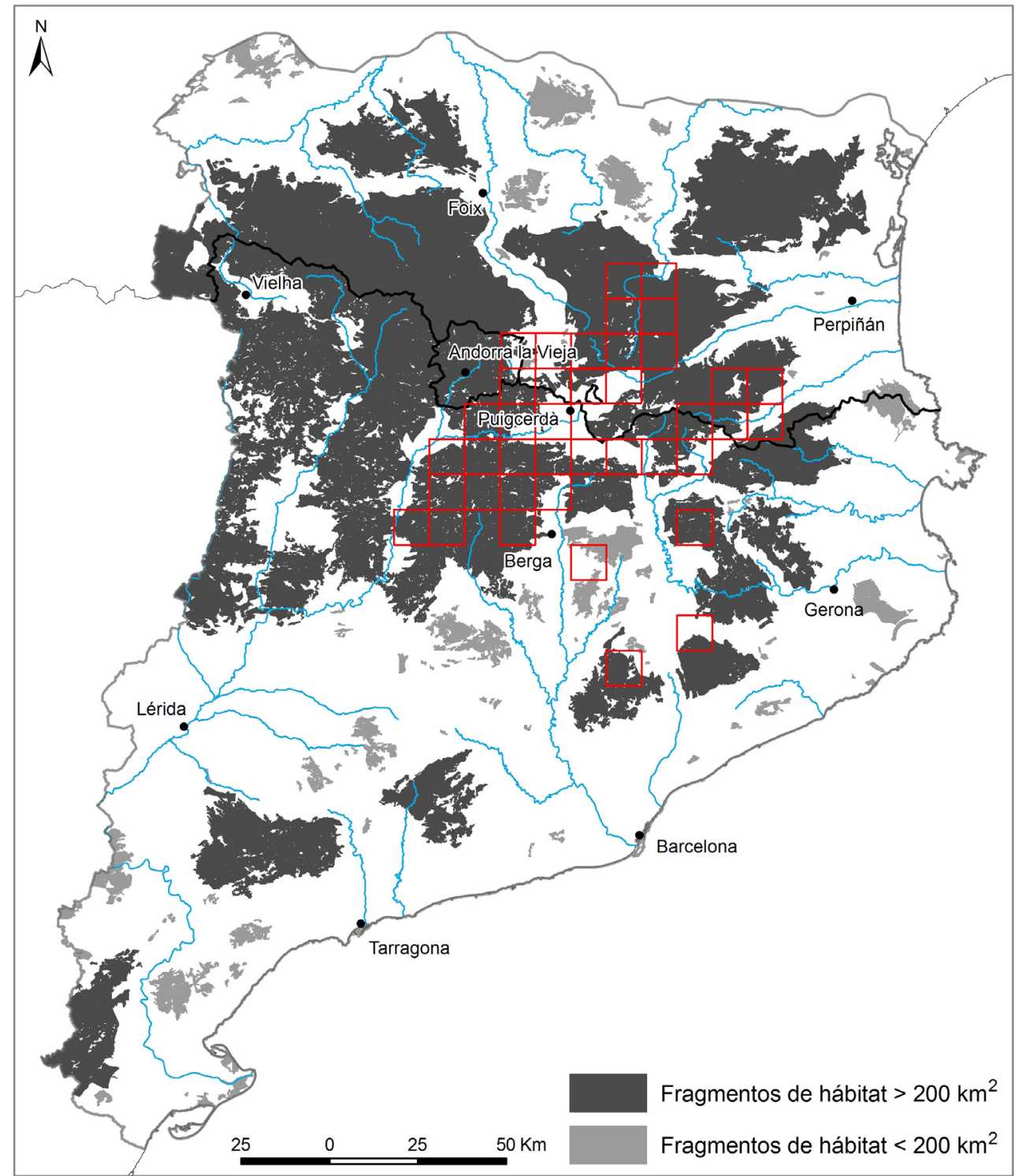

Figura 3: Fragmentos de hábitat adecuado para la presencia del lobo clasificados según su extensión. Las cuadrículas rojas indican presencia de lobo en algún momento desde 2003 hasta 2011. Fuente: Elaboración propia

Figure 3: Suitable habitat patches for wolf presence classified according to their extension. Red squares indicate presence of wolf at some time since 2003 to 2011.

$12 \mathrm{Km}$, se ha utilizado el umbral de $12 \mathrm{Km}$ para establecer el valor máximo de coste de adecuación en relación con la distancia a los cursos de agua (Tabla 2), dado que el uso de umbrales de distancia a determinados elementos del paisaje para evaluar su impacto en la distribución de las especies es de uso común en este tipo de investigaciones (Liu \& Li, 2008; Rabinowitz \& Zeller, 2010).

Los estudios realizados sobre la presencia del lobo en Galicia también han determinado que la altitud es un factor clave en la distribución de esa especie (Llaneza et al., 2012). La probabilidad de presencia del lobo en el territorio aumenta progresivamente con la altitud. El animal rehúye las zonas más bajas debido a la mayor densidad de población y presencia de la actividad humana, produciéndose la máxima probabilidad de presencia del lobo entre los 1000 y los 2000 metros, cota a partir de la cual las posibilidades de encontrar lobos disminuye nuevamente. Por tanto, se ha seguido ese criterio a la hora de asignar los valores de coste de fricción a los distintos intervalos de la variable altitud (Tabla 2).

A pesar de que no es un animal muy tímido, el lobo procura evitar los sitios excesivamente ruidosos y frecuentados, como son las grandes áreas urbanas y las infraestructuras de movilidad. La presencia de áreas habitadas es una de las principales variables que condicionan la presencia del lobo en el territorio. Los estudios realizados 
en el noroeste de la Península Ibérica corroboran que la frecuencia de lobos aumenta a medida que disminuye la densidad de viviendas y la densidad de población (Cayuela, 2004; Llaneza et al., 2012). En la asignación de valores de fricción para la variable "distancia a las áreas urbanas" se ha considerado que la presencia de lobos puede aumentar a medida que crece la distancia respecto a las áreas urbanas de extensión igual o superior a las 15 ha (superficie media de los núcleo urbanos más pequeños). Como en el caso de la distancia a las corrientes de agua se ha tomado los $12 \mathrm{Km}$ (distancia máxima diaria recorrida por el lobo) como el umbral a partir del cual la perturbación causada por la existencia de áreas urbanas es mínima (Tabla 2).

Tabla 2: Variables utilizadas en el cálculo del mapa de coste de fricción del lobo. Para los ríos se ha elegido la red fluvial permanente; para las carreteras solo se ha considerado la red viaria básica, formada por autovías, autopistas, vías desdobladas y red básica comarcal.

Table 2: Variables used in the wolf friction map. For rivers, has been chosen the permanent watercourses; for roads only has been considered the basic road network, formed by highways, two-line roads and basic regional roads.

\begin{tabular}{|c|c|c|c|c|c|c|c|}
\hline \multicolumn{2}{|c|}{ Espacios naturales protegidos } & \multicolumn{2}{|c|}{$\begin{array}{c}\text { Distancia a las áreas } \\
\text { urbanas }\end{array}$} & \multicolumn{2}{|c|}{$\begin{array}{l}\text { Distancia } \\
\text { a las carreteras }\end{array}$} & \multicolumn{2}{|c|}{$\begin{array}{c}\text { Distancia } \\
\text { al ferrocarril }\end{array}$} \\
\hline Clasificación & $\begin{array}{l}\text { Valor } \\
\text { coste }\end{array}$ & $\mathbf{K m}$ & $\begin{array}{l}\text { Valor } \\
\text { de coste }\end{array}$ & $\mathbf{K m}$ & $\begin{array}{l}\text { Valor de } \\
\text { coste }\end{array}$ & $\mathbf{K m}$ & $\begin{array}{c}\text { Valor } \\
\text { de coste }\end{array}$ \\
\hline Espacio protegido & 2 & 0 a 3 & 9 & 0 a 2 & 10 & 0 a 2 & 6 \\
\hline \multirow[t]{4}{*}{ Espacio no protegido } & 6 & 3 a 6 & 7 & 2 a 4 & 8 & 2 a 4 & 4 \\
\hline & & 6 a 10 & 5 & 4 a 8 & 6 & $>4$ & 2 \\
\hline & & 10 a 12 & 3 & 8 a 12 & 2 & & \\
\hline & & $>12$ & 1 & $>12$ & 1 & & \\
\hline
\end{tabular}

\begin{tabular}{|c|c|c|c|c|c|}
\hline \multicolumn{2}{|l|}{ Tipología de hábitats } & \multicolumn{2}{|c|}{ Distancia a los ríos } & \multicolumn{2}{|c|}{ Altitud (m) } \\
\hline Clasificación & $\begin{array}{c}\text { Valor } \\
\text { de coste }\end{array}$ & Km & $\begin{array}{c}\text { Valor } \\
\text { de coste }\end{array}$ & Intervalo & $\begin{array}{c}\text { Valor } \\
\text { de coste }\end{array}$ \\
\hline Cuerpos de agua dulce & 3 & 0 a 3 & 1 & $0-500$ & 6 \\
\hline Cuerpos de agua salobre o salina & 9 & 3 a 5 & 2 & $500-1000$ & 2 \\
\hline Canales, presas y embalses & 9 & 5 a 12 & 3 & $1000-2000$ & 1 \\
\hline Llanuras costeras arenosas o limosas & 7 & $>12$ & 5 & $>2000$ & 3 \\
\hline Matorrales y herbazales salinos & 7 & & & & \\
\hline Playas & 9 & & & & \\
\hline Roquedos litorales & 9 & & & & \\
\hline Roquedos de interior & 2 & & & & \\
\hline Landas y matorrales & 2 & & & & \\
\hline Prados y pastos & 1 & & & & \\
\hline Bosques & 1 & & & & \\
\hline Hábitats inundados & 6 & & & & \\
\hline Turberas & 2 & & & & \\
\hline Canchales & 8 & & & & \\
\hline Glaciares y nieves permanentes & 5 & & & & \\
\hline Cultivos herbáceos y leñosos & 2 & & & & \\
\hline Plantaciones de árboles & 3 & & & & \\
\hline Parques urbanos y jardines & 10 & & & & \\
\hline Ciudades, pueblos y áreas industriales & 10 & & & & \\
\hline Campos abandonados, y áreas ruderales & 5 & & & & \\
\hline Áreas quemadas o taladas & 9 & & & & \\
\hline
\end{tabular}

Fuente: Elaboración propia. 
En sentido contrario, los espacios naturales protegidos han sido ponderados favorablemente al estar reguladas o prohibidas las actividades humanas susceptibles de ejercer una perturbación negativa sobre la presencia del lobo en el territorio (Tabla 2).

Por otra parte, las grandes carreteras constituyen una clara barrera para las poblaciones del lobo y, probablemente, aíslen biogeográficamente poblaciones anteriormente bien conectadas. De hecho, estudios efectuados sobre las poblaciones de lobos del noroeste ibérico indican que el trazado de la autopista AP-9, probablemente ha separado en dos grupos la antigua población de lobos existente en el área atravesada por la vía rápida de comunicación (Rodríguez-Freire \& Crecente-Maseda, 2008). Otros autores advierten de que la probabilidad de encontrar lobos en paisajes humanizados aumenta a medida que crece la distancia respecto a las vías de comunicación principales y disminuye la densidad de carreteras por kilómetro cuadrado (Cayuela, 2004; Eggermann et al., 2011; Llaneza et al., 2012).

El atropello del lobo en las carreteras representa más del 50\% de las causas de mortalidad del animal (Mech, 1989; Llaneza et al., 2004). Un estudio sobre la distribución del lobo en Wisconsin (Thiel, 1985), llegó a la conclusión de que los lobos seleccionan territorios con densidades de tránsito bajas. Sin embargo, cuando los territorios de baja densidad de carreteras ya están ocupados por otros individuos de la misma especie, los lobos se dispersan hacia zonas de densidad viaria más elevada, aumentando así cada vez más su tolerancia a la presencia de vías de comunicación (Landry, 2004).

A pesar de que actualmente su tolerancia al ruido ha aumentado, en el pasado el ruido molesto que originaban los trenes fue la causa de que los lobos se alejaran de muchas poblaciones a mediados del siglo XIX y comienzos del XX (Manent, 2004).

En el caso de la distancia a las carreteras se ha utilizado también el valor ya indicado de $12 \mathrm{Km}$ como umbral (Tabla 2) para considerar su impacto como inapreciable a partir de esta distancia.

\section{Resultados}

El mapa de adecuación del hábitat del lobo (Figura 2) muestra que los espacios más adecuados para el establecimiento del lobo coinciden con las principales masas boscosas que se localizan en las zonas montañosas, justamente los lugares menos humanizados. Destaca el alto grado de adecuación del Alto Pirineo catalán, pero sobre todo de los Pirineos franceses: las zonas del parque natural regional de los Pirineos Catalanes, el parque natural regional de los Pirineos del Ariège y el macizo de las Corbières.

En cambio, los espacios menos propicios para el establecimiento del lobo se localizan en las zonas urbanizadas, sobre todo en la llanura costera, y en las planicies litorales como la Plana del Empordà y la Plana del Rosselló, y en menor proporción en los llanos o depresio- nes del interior de las tierras catalanas. Tampoco son óptimos para el asentamiento y desplazamiento del lobo los principales corredores de infraestructuras viarias como la zona de la Depresión Prelitoral.

Los lugares que menor valor de coste de adecuación acumulan constituyen el hábitat potencial del lobo, las áreas más propicias para su reproducción y hallazgo de recursos (Figura 3). Se observa claramente en el mapa que las teselas más favorables están situadas en las principales cadenas montañosas: la Cordillera Prelitoral Catalana, los Pirineos y los Prepirineos y, en menor proporción, en ciertas áreas de la Cordillera Litoral Catalana y de las planicies interiores.

Por otra parte, dentro de las zonas óptimas para la reproducción del lobo, se han diferenciado los espacios donde el animal podría formar manadas de forma estable y aquellas zonas más pequeñas donde sólo podría llevar a cabo incursiones puntuales o facilitar la dispersión de individuos solitarios hacia espacios de mayor entidad, a menos que los fragmentos menores presentaran un buen estado de la conectividad paisajística con el resto de fragmentos cercanos.

Una manada de lobos necesita un espacio para satisfacer sus necesidades de dimensión inversamente proporcional a la disponibilidad de alimentos. Algunos estudios revelan que, de media, el espacio mínimo para albergar al lobo ibérico está alrededor de los $200 \mathrm{~km}^{2}$ (Vilà et al., 1990); mientras que según Landry (2004) las manadas de lobos italianos recientemente llegados al Mercantour francés también utilizan un territorio de aproximadamente $200 \mathrm{~km}^{2}$ para satisfacer sus necesidades vitales.

De esta manera, a partir del mapa de adecuación del hábitat se han seleccionado aquellas áreas de extensión superior a los $200 \mathrm{~km}^{2}$ como las áreas susceptibles de albergar poblaciones estables de lobos (Figura 3). La mayor parte de estos lugares se localizan en el área de los Pirineos y Prepirineos, donde se concentran fragmentos de gran extensión, contiguos o muy cercanos entre ellos. Se destacan territorios como el parque natural regional de los Pirineos Catalanes, el parque natural regional de los Pirineos de l'Ariege y el macizo de las Corbières (en la vertiente francesa) o el parque natural del Alto Pirineo, el Alta Garrotxa, la Sierra Cavallera, la Sierra del Cadí, el Port del Compte, la Sierra de Boumort, el Montsec o el Macizo de Beret.

Así mismo, también se localizan algunos fragmentos superiores a los $200 \mathrm{~km}^{2}$ en sierras pertenecientes a la Cordillera Prelitoral Catalana (los Ports de Beseit, la Sierra del Montsant y las Montañas de Prades, la Sierra de Miralles y el Montmell, Sant Llorenç del Munt i l'Obac, el Montseny y las Guillerías). Son fragmentos de hábitat propicios para el lobo pero sin continuidad física entre ellos, pero en cualquier caso, con condiciones óptimas para albergar poblaciones estables.

Finalmente, una serie de fragmentos de hábitat de menor extensión se encuentran situados en distintos puntos del centro de Cataluña, la Cordillera Litoral y el extremo oriental del Pirineo. 


\section{Discusión y conclusiones}

Los lobos disponen de una gran extensión de hábitat potencialmente óptimo para su establecimiento y dispersión a lo largo del área estudiada, puesto que presentan una gran adaptabilidad a diferentes tipologías de ambientes. Los resultados obtenidos a partir del mapa de adecuación del hábitat para el lobo constatan que un tercio del territorio analizado constituye un hábitat o ambiente adecuado para esta especie.

El abandono de las actividades agrarias que tuvo lugar desde finales del siglo XIX y especialmente a partir de la segunda mitad del siglo XX, ha propiciado la recuperación de la superficie forestal, que constituye masas densas y continuas especialmente en la mitad norte del área de estudio. Debido a esta dinámica territorial, las zonas ideales para el lobo se localizan en las principales cadenas montañosas y masas forestales, sobre todo en el área de los Pirineos y Prepirineos y, en menor proporción, en las Cordilleras Prelitoral y Transversal catalanas.

Los fragmentos de hábitat de notables dimensiones son muy numerosos. Cerca del $90 \%$ de los parches de hábitat potencial presentan unas dimensiones superiores a los $200 \mathrm{~km}^{2}$, mientras que el $10 \%$ restante no supera esta superficie. Con ello, casi la totalidad de los fragmentos son susceptibles de albergar manadas estables o manadas de lobos si se dan las condiciones de reproducción necesarias.

Si se recupera el flujo de lobos solitarios en el área estudiada y se forman manadas estables, dada la característica de contigüidad o proximidad entre los principales fragmentos de hábitat, el animal podría dispersarse a lo largo de buena parte del territorio. El único obstáculo que debería hacer frente son las infraestructuras lineales de movilidad terrestres. A pesar de ello, es evidente que -en menor o mayor medida- este mamífero podría superar las grandes vías de comunicación, tal y como ha hecho a lo largo de su dispersión desde los Apeninos centrales hasta los Pirineos orientales, a través de áreas tan densamente pobladas como el norte de Italia o el arco mediterráneo francés.

El elevado grado de disponibilidad de ambientes propicios para el cánido haría posible la presencia del mamífero en muchas zonas de Cataluña, Andorra o el sector oriental de los Pirineos franceses. Asimismo, el lobo podría establecerse en los fragmentos de hábitat de mayor dimensión, mientras que todos aquellos que no superan una superficie de $200 \mathrm{~km}^{2}$ mayormente podrían ser utilizados para su dispersión o para incursiones puntuales de lobos solitarios. Además, este depredador puede realizar incursiones donde haya abundancia de presas, a pesar de que sean áreas ajenas a su ambiente (Landry, 2004). De hecho, para determinar con exactitud si las manadas de lobo pueden o no establecerse en los fragmentos de hábitat inferiores a $200 \mathrm{~km}^{2}$ deberíamos realizar un estudio de conectividad entre dichos fragmentos y el resto de parches de hábitat cercanos.

Por otra parte, las muestras que hasta ahora se han recogido en la zona de estudio indican que el lobo frecuenta la zona del Cadí-Moixeró, en Cataluña, mientras que en Francia se localiza en el parque natural regional de los Pirineos Orientales (Figura 1), ya que según Lampreave et al. (2011) cuentan con una gran disponibilidad de presas naturales para el lobo, como liebres (Lepus europaeus), jabalíes (Sus scrofa), corzos (Capreolus capreolus), muflones (Ovis orientalis), ciervos (Cervus elaphus) y rebecos pirenaicos (Rupicapra pyrenaica). Estos lugares coinciden con la localización de los fragmentos más extensos de hábitat que se han obtenido en este análisis (Figura 3).

En este sentido, cabe destacar el papel ecoregulador del lobo puesto que elimina los animales viejos o débiles, dejando sólo los más adaptados al medio, evitando epidemias entre las poblaciones de ungulados y mejorando la salud general de los ecosistemas. Además, téngase en cuenta que recientemente se han reintroducido especies extinguidas al Pirineo como la marmota (Marmota) o el muflón (Ovis orientalis), depredadas en estado natural por el lobo. El éxito de esta introducción depende en buena medida de la presencia de un animal que pueda regular dichas poblaciones.

Así pues, si vuelve a aumentar el flujo de lobos procedentes de Francia y se dan las condiciones reproductivas necesarias, se podría establecer un macrogrupo nuclear en la zona del Cadí-Moixeró y de los Pirineos Orientales (Francia) que asegurara el mantenimiento natural de la población existente, así como la dispersión de nuevos individuos hacia la periferia de estos espacios. La protección de la que disfruta el lobo a escala europea (mediante convenios, directivas y tratados internacionales), francesa $\mathrm{y}$, en un futuro inminente, catalana, favorece el establecimiento y la dispersión del predador a lo largo de los Pirineos franceses y en todo el territorio catalán.

La presencia del lobo de nuevo en los Pirineos no deja indiferente al conjunto de la población, ya sea para alegrarse o lamentar su llegada. El conflicto entre los humanos y el lobo seguirá siendo una realidad en la tensa relación entre el animal y los ganaderos debido a los ataques constantes del predador a la ganadería extensiva. De hecho, en el área de estudio ya se han registrado numerosos ataques a rebaños por parte de lobos y perros asilvestrados, puesto que en el sector pirenaico del área de estudio abunda la ganadería extensiva y trashumante. En este sentido, y debido a que las poblaciones de lobo todavía no son estables en la zona, los ataques al ganado se atribuyen en la inmensa mayoría de los casos a perros y no a lobos (Lampreave et al., 2011). En Italia, donde las poblaciones de Canis lupus italicus son estables, Boitani (1983) estima que en las zonas donde hay una densidad media de 1 lobo/100 $\mathrm{km}^{2}$ existen $150-310$ perros sueltos y 24-82 perros asilvestrados/100 $\mathrm{km}^{2}$. A pesar de que los perros sueltos y asilvestrados suponen un problema numérico mayor al de los lobos, para garantizar la convivencia entre biodiversidad y actividades económicas habría que implementar métodos de prevención tradicionales como los rebaños cercados o la vigilancia con mastines, así como seguir aplicando medidas compensatorias cuando el daño al ganado esté causado por lobos. 
Por otro lado, la presencia del lobo supone un incentivo para el turismo amante de la naturaleza y el resto de actividades económicas que lo rodean, favoreciendo el desarrollo en las áreas rurales y mejorando la visión que las sociedades locales tienen hacia el lobo. Esto ya sucedió en el Parque Nacional del Mercantour, en Francia, donde la recuperación del predador ha supuesto un incremento en la presencia de visitantes (González-Prat, 2002). En este sentido, cabe destacar los esfuerzos que se llevan a cabo desde las administraciones públicas de sensibilización y divulgación mediante material didáctico sobre el lobo y su presencia que se reparte a las escuelas y a los turistas de la zona, así como charlas y cursos en escuelas y universidades, asociaciones ganaderas y de pastores, jornadas o congresos (Lampreave et al., 2011).

En conclusión, la zona estudiada muestra unas condiciones de disponibilidad de hábitat suficientemente óptimas para asegurar el establecimiento y la dispersión del cánido. Los resultados obtenidos constatan que en la mitad este de los Pirineos, tanto en las vertiente francesa como ibérica, y en los Prepirineos el establecimiento definitivo del lobo podría ser una realidad inminente si la entrada de lobos procedentes de Italia vuelve a los niveles alcanzados pocos años atrás. Frente a esta realidad es imprescindible que los esfuerzos de gestión de la especie centren sus esfuerzos en lograr una buena convivencia entre lobo, ganadería y turismo.

\section{Agradecimientos}

Una parte de este trabajo constituyó el Trabajo Final de Máster de Carla Garcia en el Programa de Doctorado de Cambio Ambiental de la Universitat de Girona. A David Camps, del Servicio de Biodiversidad y Protección de los Animales del Departament d'Agricultura, Ramaderia, Pesca, Alimentació i Medi Natural (DARPAM) de la Generalitat de Catalunya por su información sobre la presencia actual del lobo en Cataluña. A Pere Pons del Instituto de Medio Ambiente de la Universitat de Girona por sus comentarios sobre una primera versión del trabajo.

\section{Referencias}

Acevedo, P., Real, R. \& C. Gortazar, 2011. Favorabilidad ecogeográfica para el corzo: Distribución y abundancia. Pirineos, 166: 9-27. http://dx.doi.org/10.3989/pirineos.2011. 166001

Blanco, J. C. \& Cortés, Y., 2002. Ecología, censos, percepción y evolución del lobo en España: Análisis de un conflicto. Sociedad Española para la Conservación y Estudio de los Mamíferos (SECEM), 165 pp., Málaga.

Blanco, J. C., Sáenz de Buruaga, M. \& Llaneza, L., 2007. Canis lupus Linneaus, 1758. En Atas y Libro Rojo de los mamiferos terrestres de España, 272-274. L.J. Palomo, J. Gisbert y J.C. Blanco (eds.). Sociedad Española para la Conservación y Estudio de los Mamíferos (SECEM), 586 pp., Málaga.

Boitani, L., 1983. Wolf and dog competition in Italy. Acta Zool. Fennica, 174: 259-264.
Butchart, S. H., Walpole, M., Collen, B., Van Strien, A., Scharlemann, J. P., Almond, R. E., ... \& Watson, R., 2010. Global biodiversity: indicators of recent declines. Science, 328 (5982): 1164-1168. http://dx.doi.org/10.1126/science. 1187512

Cayuela, L., 2004. Habitat evaluation for the Iberian wolf Canis lupus in Picos de Europa National Park, Spain. Applied Geography, 24 (3): 199-215. http://dx.doi.org/10.1016/j. apgeog.2004.04.003

Chapron, G., Kaczensky, P., Linnell, J. D., Von Arx, M., Huber, D., Andrén, H., ... \& Nowak, S., 2014. Recovery of large carnivores in Europe's modern human-dominated landscapes. Science, 346: 1517-1519. http://dx.doi.org/10.1126/ science. 1257553

Echergaray, J., \& Vilà, C., 2010. Noninvasive monitoring of wolves at the edge of their distribution and the cost of their conservation. Animal Conservation, 13: 157-161. http:// dx.doi.org/10.1111/j.1469-1795.2009.00315.x

Eggermann, J., da Costa, G. F., Guerra, A. M., Kirchner, W. H. \& Petrucci-Fonseca, F., 2011. Presence of Iberian wolf (Canis lupus signatus) in relation to land cover, livestock. Mammalian Biology - Zeitschrift Für Säugetierkunde, 76 (2): 217-221. http://dx.doi.org/10.1016/j.mambio.2010.10.010

ETC/SIA (European Topic Centre for Spatial Information and Analysis), 2013. Corine Land Cover 2006 raster data. Raster data on land cover for the CLC2006 inventory, Versión 17, 100 metros de resolución, Dinamarca: European Environment Agency (EEA).

Fourli, M.,1999. Compensation for Damage Caused by Bears and Wolves in the European Union. Luxemburg. DG XI European Comunities.

Franklin, J., 2009. Mapping Species Distributions. Spatial Inference and Prediction. Cambridge University Press, 344 pp., Cambridge.

González-Prat, F., 2002. El retorn del 1lop (Canis lupus, L., 1758) als Pirineus. (Reflexions sobre conservació de la natura i el món pastorívol a la muntanya). Annals Del Centre d'Estudis Comarcals del Ripollès, 2000-2001: 301-312.

Gortázar, C., Herrero, J., Villafuerte, R. \& Marco, J., 2000. Historical examination of the status of large mammals in Aragon, Spain. Mammalia, 64 (4): 411-422. http://dx.doi. org/10.1515/mamm.2000.64.4.411

Grau, J.M., Puig, R. \& Ruiz-Olmo, J., 1990. Persecución del lobo (Canis lupus L., 1758) en Girona (NE Ibérico) durante los siglos XVIII y XIX: ejemplo de utilización de datos de archivo. Miscelánea Zoológica, 14: 217-223.

Gurrutxaga, M., Lozano, P. J. \& del Barrio, G., 2010. GIS-based approach for incorporating the connectivity of ecological networks into regional planning. Journal for Nature Conservation, 18 (4): 318-326. http://dx.doi.org/10.1016/j.jnc. 2010.01.005

Hoffmann, M., Hilton-Taylor, C., Angulo, A., Böhm, M., Brooks, T. M., Butchart, S. H., ... \& Collett, L. C., 2010. The impact of conservation on the status of the world's vertebrates. Science, 330: 1503-1509. http://dx.doi.org/ $10.1126 /$ science. 1194442

Landry, J.M., 2004. El lobo. Editorial Omega, 248 pp., Barcelona.

Lampreave, G., Ruiz-Olmo, J., Garcia-Petit, J., López, J. M., Bataille, A., Sastre, N., Francino, O. \& Ramírez, O., 2011. El lobo vuelve a Cataluña: historia del regreso y medidas de conservación. Quercus, 302: 16-25.

Liu, X. \& Li, J., 2008. Scientific solutions for the functional zoning of nature reserves in China. Ecological Modelling, 215 (1-3): 237-246. http://dx.doi.org/10.1016/j.ecolmodel.2008.02.015

Llaneza, L., Alvares, F., Ordiz, A., Sierra, P. \& Uzal, A., 2004. Distribución y aspectos poblacionales del lobo ibérico en la Provincia de Ourense. Ecología, 18: 227-238.

Llaneza, L., López-Bao, J. V. \& Sazatornil, V., 2012. Insights into wolf presence in human-dominated landscapes: the 
relative role of food availability, humans and landscape attributes. Diversity and Distributions, 18 (5): 459-469. http:// dx.doi.org/10.1111/j.1472-4642.2011.00869.x

Manent, A., 2004. El llop a Catalunya: memòria, llegenda i història. Pagès Editors, 232 pp., Lleida.

Mech, L.D., 1989. Wolf population survival in area of high road density. American Midland Naturalist, 121: 387-389.

Mech, L.D., 1995. The Challenge and opportunity of recovering Wolf Populations. Conservation Biology, 9 (2): 1-9.

Núñez-Quirós, P., García-Lavandera, R. \& Llaneza, L., 2007. Análisis de la distribución histórica del lobo (Canis lupus) en Galícia: 1850, 1960 y 2003. Ecología, 21: 195-206.

Poulle, M. L., Houard, T. \& Dahier, T., 1995. Le suivi des loups dans le Parc National du Mercantour. Bulletin Mensuel de l'Office National de la Chasse, 201: 36-43.

Rabinowitz, A. \& Zeller, K. A., 2010. A range-wide model of landscape connectivity and conservation for the jaguar, Panthera onca. Biological Conservation, 143 (4): 939-945. http://dx.doi.org/10.1016/j.biocon.2010.01.002

Rico, M. \& Torrente, J. P., 2000. Caza y rarificación del lobo en España: investigación histórica y conclusiones biológicas. Galemys, 12: 163-179.

Rodríguez-Freire, M. \& Crecente-Maseda, R., 2008. Directional connectivity of wolf (Canis lupus) populations in northwest Spain and anthropogenic effects on dispersal patterns.
Environmental Modeling \& Assessment, 13 (1): 35-51. http://dx.doi.org/10.1007/s10666-006-9078-y

Sáenz de Buruaga, M., Campos, M. A., Arberas, E. \& Onrubia, A., 2000. Últimos datos sobre el lobo (Canis lupus) en el País Vasco y Navarra. Galemys, 12: 149-162.

Salvatori, V. \& Linnell, J., 2005. Report on the conservation status and threats for wolf (Canis lupus) in Europe. Consejo de Europa. Estrasburgo.

Suau, A., 2012. Model d'adequació de l'hàbitat del llop (Canis lupus) a Catalunya. Dipòsit Digital de Documents de la UAB.

Thiel, R.P., 1985. Relationship between road densities and wolf habit suitability in Wisconsin. American Midland Naturalist, 113: 404-407.

Velez-Liendo, X., Adriaensen, F. \& Matthysen, E., 2014. Landscape assessment of habitat suitability and connectivity for Andean bears in the Bolivian Tropical Andes. Ursus, 25 (2): 172-187. http://dx.doi.org/10.2192/URSUS-D-14-00012.1

Vilà, C., Urios, V. \& Castroviejo, J., 1990. Ecología del lobo en la Cabrera (León) y la Carballeda (Zamora). En Blanco, J.C., y Reig. S. (eds). El lobo (Canis lupus) en España. Situación, problemática y apuntes sobre su ecología, 95-108. Madrid, Colección Técnica I.C.O.N.A.

Zeller, K. A., McGarigal, K. \& Whiteley, A. R., 2012. Estimating landscape resistance to movement: a review. Landscape Ecology, 27: 777-797. http://dx.doi.org/10.1007/s10980-012-9737-0 